\title{
Percepción y conocimiento de los docentes universitarios sobre los procesos investigativos universitarios: estudio de caso
}

\author{
Alejandro Valencia-Arias' \\ Daniela Morales-Zapata" \\ Laura Vanegas-Rendón ${ }^{\text {III }}$ \\ Martha Luz Benjumea-Ariasiv
}

I- Instituto Tecnológico Metropolitano, Medellín, Colombia.

\section{Resumen}

Dentro de las tareas que tienen actualmente las universidades e instituciones de educación superior está la de fortalecer los nexos entre la enseñanza y la investigación, promoviendo en los estudiantes y docentes el interés por la generación de conocimiento desde las aulas y los diferentes espacios destinados para ello. El objetivo de la presente investigación es el de conocer las percepciones de los docentes en cuanto a los procesos de investigación llevados a cabo por la Universidad, desde la participación de los mismos docentes y estudiantes. A través de la aplicación de entrevistas a profundidad a docentes de la Facultad de Ciencias Económicas y Administrativas de Uniminuto, se pretendió indagar sobre aspectos como: conocimiento, promoción, percepción, expectativa y motivación, en cuanto a dichos procesos de investigación. Los principales puntos extraídos del análisis de estas encuestas recaen en la falta de divulgación de estos procesos y la falta de brindar motivación por parte de las directivas de la universidad, hacia los diferentes miembros de la comunidad universitaria. Además, fue posible evidenciar un problema dentro de la institución en cuanto a la insatisfacción de las expectativas que los profesores y estudiantes tienen sobre los procesos investigativos; lo cual lleva a que sea necesaria una reestructuración y un enfoque diferente de algunas líneas, complaciendo así la petición más común, orientada hacia la apertura del conocimiento al exterior y el compartir con otras comunidades investigativas del país y del extranjero de ser posible.

\section{Palabras clave}

Enseñanza - Procesos de investigación - Universidad - Generación de conocimiento. 


\title{
Perception and knowledge of university professors about university research: a case study
}

\author{
Alejandro Valencia-Arias' \\ Daniela Morales-Zapata" \\ Laura Vanegas-Rendón"II \\ Martha Luz Benjumea-Arias ${ }^{1}$
}

\begin{abstract}
One of the current tasks of universities and higher educational institutions is to strengthen the links between teaching and research, promoting students' and professors' interest in the generation of knowledge from the classes and different spaces. The purpose of this research is to understand the perceptions of professors regarding the research processes conducted by the university, considering the participation of professors and students. Through in-depth interviews with professors of Facultad de Ciencias Económicas $y$ Administrativas de Uniminuto, aspects related to research processes, such as knowledge, promotion, perception, expectation, and motivation, were examined. The main findings of the analysis of these interviews coincide in the lack of dissemination of these processes and the lack of promotion of motivation by university policies to the different members of the university community. In addition, it was possible to evidence a problem within the institution related to not meeting the expectations that professors and students have about research processes, which results in the need to restructure and have a different approach to some lines thus meeting the most frequent request -, oriented to opening and sharing knowledge with other research communities in the country and abroad if it is possible.
\end{abstract}

\section{Keywords}

Teaching - Research processes - University - Knowledge generation.

I- Instituto Tecnológico Metropolitano, Medellín, Colombia

Contact: jhoanyvalencia@itm.edu.co

II- Universidad Nacional de Colombia, Medellín, Colombia.

Contact: dmoralesz@unal.edu.co

III- Universidad de los Andes, Bogotá, Colombia.

Contact: lacvanegasre@gmail.com

IV- Instituto Tecnológico Metropolitano,

Medellín, Colombia.

Contact: marthabenjumea@itm.edu.co 


\section{Introducción}

\section{Estado del arte}

Durante el siglo XIX y principios del siglo $\mathrm{XX}$, las universidades europeas fueron centros líderes en investigación y educación. Estas universidades atrajeron científicos, académicos $\mathrm{y}$ estudiantes de todo el mundo para llevar a cabo procesos de investigación de vanguardia $\mathrm{y}$ aprender de las principales autoridades de la época. Por su parte, para universidades de Estados Unidos como Harvard y Yale, Europa fue un modelo a seguir. Sin embargo, el panorama de la educación ha cambiado y desde el fin de la Segunda Guerra Mundial, las universidades de Estados Unidos constituyen la mayoría de las instituciones líderes en el mundo para la investigación y la educación (ARAI et al., 2007). Su sistema de investigación ahora se ha caracterizado por la fusión de la educación y la investigación de vanguardia, mientras que Europa sigue un modelo de investigación tradicional, el cual se centra en la investigación en institutos especiales más que en universidades (ASSOCIATION..., 2011).

Además, las economías que anteriormente estaban basadas en la industria manufacturera, ahora son economías basadas en conocimiento que dependen especialmente de la investigación científica y la fuerza laboral capacitada. Actualmente la competencia no está enlazada a la capacidad industrial o al acceso a los recursos naturales directamente, sino a trabajadores calificados, a la propiedad intelectual y al conocimiento (ARAI et al., 2007).

\section{Futuro y retos de la investigación}

Rip (2011) plantea que el reto de la ciencia es su recontextualización en la sociedad. Este autor también plantea que las universidades son lugares en donde se puede llevar a cabo esta recontextualización. Este concepto de recontextualización se basa en cierta convergencia de un régimen emergente de la ciencia estratégica, de la modernización reflexiva visible en las instituciones de ciencia y de la producción de conocimiento. La aparición de este nuevo régimen de ciencia estratégica ha sido clave del cambio de las universidades de investigación y el sistema de investigación en los últimos 30 años (RIP, 2011). Este régimen ofrece mercados para la investigación estratégica, la cual Irvine y Martin (1984) definen como investigación básica cuyo objetivo es producir una amplia base de conocimientos y que la Association of American Universities (2011) complementa con que se enfoca a una compresión más completa del objeto de estudio sin aplicaciones específicas en mente. Desde principios de los años ochenta, la investigación estratégica es un concepto que ha permeado el mundo de la ciencia. Por una parte, la alianza formada entre los políticos que realmente están preocupados por el futuro de la ciencia y por otra parte, una nueva élite de científicos que prometen contribuir a la creación de riqueza y sostenibilidad están dominando la política y financiación de la ciencia (RIP, 2011).

Arai y otros autores (2007) resaltan que el futuro de las economías se encuentra ligado al futuro de las universidades de investigación y que éstas, a su vez, deben producir estudiantes de maestrías y doctorados, atraer talento de todo el mundo y entrenar recursos humanos. También señalan que la situación para una universidad de investigación depende de la etapa de desarrollo, tanto científica como económica, en la que se encuentre el país en el que está ubicada la universidad y que la misión más importante de las universidades de investigación en las naciones desarrolladas es promover la investigación creativa individual, la cual, es la base para apoyar a la industria innovadora; pero para esto es necesaria una infraestructura donde docentes y estudiantes graduados se puedan inspirar para desarrollar ideas creativas.

Por otra parte, las universidades de investigación en lugar de inclinarse por una tendencia de volverse más especializadas, se deberían enfocar en integrar la enseñanza y 
la investigación con el objetivo de crear una verdadera comunidad académica. Del éxito y fortalezas de las universidades de investigación depende en gran medida el desarrollo económico y las oportunidades para los ciudadanos de un país. Las universidades de investigación tienen un rol crítico, no sólo para que los estudiantes basen sus decisiones y opiniones en evidencia existente, sino para que también permitan desarrollar cierto entusiasmo por una carrera de investigación. Además, las universidades deben actuar internacionalmente, deben tener autonomía para convertirse en innovadores y responsables del cambio, al igual que desarrollar sociedades estructuradas con la comunidad de negocios y con los socios potenciales (ARAI et al., 2007).

Otros autores resaltan que existe una preocupación constante sobre el reclutamiento de estudiantes talentosos en educación de posgrado, pues hay barreras para continuar con estudios de posgrado, como los altos costos y la baja propensión por parte de las instituciones a brindar apoyo financiero en términos de matrículas y exenciones. Este caso se presenta especialmente en estudiantes que buscan especialización en áreas como medicina, derecho y negocios. Aunque los estudiantes de doctorado están bien apoyados, a menudo se acumula una sustancial deuda financiera (HATHAWAY; NAGDA; GREGERMAN, 2002).

A pesar de los retos inherentes a continuar con una educación de posgrado, una importante porción de estudiantes de primer año de diferentes campos (Inglés, Biología, etc.) y diferentes grupos étnicos indican que esperan seguir con estudios de posgrado (HATHAWAY; NAGDA; GREGERMAN, 2002). De acuerdo a lo planteado por Alexander Astin y Helen Astin, (1992) y Hathaway, Nagda y Gregerman (2002), las experiencias de investigación en el pregrado juegan un rol muy importante en la continuación de estudios de posgrado. Por ejemplo, participar en actividades o talleres raciales o étnicos, tomar cursos interdisciplinarios, participar en proyectos de investigación e interactuar con el profesorado, son factores críticos no sólo en el éxito académico, sino en la retención de estudiantes y en que estos se enfoquen en desarrollar estudios superiores.

De acuerdo a lo planteado por la literatura, las universidades de investigación tienen retos importantes, los cuales se deberían enfocar en capitalizar su experiencia y beneficiarse del nexo enseñanza-investigación, proporcionando a sus estudiantes experiencias de investigación (WILSON et al., 2012), que le permita a la universidad permanecer enfocada en la creación y diseminación de conocimiento y al mismo tiempo fortalecer su autonomía institucional (ARAI et al., 2007); para de esta forma poder asumir los diferentes y nuevos desafíos que propone el desarrollo económico.

\section{Percepciones y efectos de la participación de estudiantes y docentes en la investigación}

Los efectos positivos de la participación de estudiantes de pregrado en experiencias de investigación están vinculados a beneficios personales y profesionales, como el incremento en el compromiso con sus estudios universitarios; clarificando y confirmando su carrera profesional, mejorando su preparación para estudios de posgrado, e incluso cambiando actitudes para aprender y trabajar como un investigador (SEYMOUR et al., 2004). La participación en procesos de investigación incentiva a que los estudiantes continúen con estudios de posgrados, especialmente en campos de ciencia, tecnología, ingeniería y matemáticas, y se logra mayor entendimiento de cómo gestionar un proyecto de investigación, alcanzar un mayor nivel de confianza en las habilidades y conciencia de lo que es la escuela de posgrado; inclusive, se desarrolla cierta expectativa en alcanzar un título de $\mathrm{PhD}$ que no se tenía antes de ingresar al pregrado (RUSSEL; HANCOCK; MCCULLOUGH, 2007). Adicionalmente, los estudiantes logran alcanzar un mayor entendimiento de la literatura científica y de sus campos de estudio, a través de la aplicación en problemas prácticos, con lo 
cual mejoran sus habilidades como la resolución de problemas, trabajo en equipo, comunicación oral y escrita y síntesis de información (WILLIS; KRUGER; KENDRICK, 2013).

La participación en investigación en el pregrado se ha sostenido como una actividad enriquecedora que aumenta la probabilidad de que los estudiantes persistan en especializarse en estudios relacionados con el campo de la ciencia. Aunque no se evidencia un estudio sistemático de su eficacia (VILLAREJO; BARLOW; KOGAN, 2008), existe una asociación entre participar en un proyecto de investigación de un profesor, realizar investigación independiente, apoyar a docentes en cursos de enseñanza y continuar o reclutarse en un programa de especialización científico o carreras afines (ASTIN; ASTIN, 1992). Es decir, este tipo de participación despierta interés en los estudiantes hacia campos científicos, incrementa la satisfacción de los alumnos con cursos de ciencias y con la experiencia institucional.

Un estudio liderado por Hathaway, Nagda y Gregerman (2002) demuestra que aquellos estudiantes que se involucran en experiencias de investigación en el pregrado son más propensos a continuar con estudios de posgrado, con actividades de investigación y utilizar al profesorado para recomendaciones de trabajo, más que aquellos que no participan de dichas experiencias; lo cual es consistente con lo planteado en la literatura de la educación superior, en la cual se sugiere que la interacción estudiantes - profesorado y la participación en investigación influencian las aspiraciones académicas de los estudiantes (ASTIN, A; ASTIN, H, 1992; PASCARELLA; TERENZINI, 1991 apud HATHAWAY; NAGDA;GREGERMAN, 2002).

La participación de docentes en experiencias de investigación influencia las creencias en sus prácticas pedagógicas, teniendo efectos en la planeación de cómo enseñar determinada ciencia, permitiéndoles desarrollar experiencias de aprendizaje más enriquecedoras, realistas, relevantes y rigurosas, que promueven el compromiso de los estudiantes con la ciencia.
Esto se complementa con que un componente importante en la enseñanza de alta calidad y los resultados de los estudiantes, se encuentran vinculados a las prácticas pedagógicas (MIRANDA; DAMICO, 2013). Incluso se afirma que los docentes que implementan prácticas pedagógicas basadas en investigación en los salones de clase, logran mejorar las habilidades en los procesos científicos de los estudiantes, influyen ciertos hábitos mentales, incrementan las habilidades para la resolución de problemas y el entendimiento de la naturaleza de la ciencia (HOFSTEIN; LUNETTA, 2004; MIRANDA; DAMICO, 2013).

De acuerdo al reporte de Boyer (WILSON et al., 2012), los estudiantes de pregrado deberían participar en tantos procesos de investigación como sea posible, atado a la creencia y percepción de que la enseñanza y la investigación pueden mejorar las experiencias de aprendizaje de los estudiantes.

Según Willis, Krueger y Kendrick (2013) las experiencias de investigación en el pregrado son valoradas por el profesorado universitario y las instituciones, debido a la creencia de que dichas experiencias despiertan un mayor interés en los estudiantes de participar en escuelas de posgrado. Estos mismos autores hacen especial hincapié en el rol que juegan tanto los docentes como el mismo sistema de reclutamiento de estudiantes de investigación liderado por las instituciones universitarias. Aunque no se ha demostrado una relación evidente entre el acompañamiento de los docentes y los resultados positivos que una experiencia investigativa pudiese tener en los estudiantes, ellos expresaron que una forma de mejorar los programas de investigación de pregrado radica en incrementar el acompañamiento de los docentes o hacer más efectiva su orientación, según Russel, Hancock y Mccullough (2007). Estos autores sospechan que el rol de tutor por parte de los docentes, reviste cierta complejidad e indica que son capaces de combinar habilidades entusiastas, interpersonales, organizacionales e investigativas, representan un papel importante en la facilitación para alcanzar resultados 
positivos. Incluso WILSON y otros autores (2012) resaltan que el acompañamiento es un factor determinante del éxito percibido o el valor de la experiencia para el estudiante.

De lo anterior se puede rescatar la importancia de incentivar los procesos investigativos en las aulas de clase y de promover la participación tanto de estudiantes como de profesores. En la revisión de literatura llevada a cabo, por ejemplo, no se encontraron producciones académicas que contemplaran percepciones de los docentes de los procesos investigativos en Colombia o que indagaran por los conocimientos que tenían estos respecto a este tema. Las publicaciones relacionadas con la investigación a nivel universitario tratan, entre otras cosas, de cartillas para el docente universitario y su proceso de mejoramiento en la calidad de la enseñanza-aprendizaje de sus estudiantes (TAMAYO; RESTREPO, 2011), de artículos sobre la importancia de la investigación en la educación superior (GONZALEZ, 2012), de encuestas sobre las percepciones de las ciencias y las tecnologías en Colombia (DAZA-CAICEDO et al., 2014; AGUIRRE, 2005), y de la promoción de la investigación por parte de las universidades en Colombia (COLOMBIA, 2005). El estudio más cercano a la investigación propuesta en el presente artículo, es el estudio de caso en seis instituciones de Educación Superior en Colombia, expuesto por Rojas (2011), en el que se indagan por las opiniones y percepciones del joven universitario sobre la investigación en sus respectivas universidades. Por lo tanto, el objetivo de la presente investigación es el de conocer las percepciones de los docentes en cuanto a los procesos de investigación llevados a cabo por la institución, desde la participación de los mismos docentes y su opinión sobre la participación de los estudiantes.

\section{Metodología}

Se aplicaron entrevistas a profundidad a docentes de la Facultad de Ciencias Económicas y Administrativas de Uniminuto, siendo estas entrevistas uno de los principales métodos para recolectar información en investigación cualitativa (LEGARD, KEEGAN; WARD, 2003). La entrevista a profundidad es concebida como una situación entre dos personas, en la que se van turnando la toma de la palabra, de manera que el entrevistador propone temas y el entrevistado trata de producir respuestas localizadas y centradas en las preguntas planteadas (CALLEJO, 2002). Con la entrevista a profundidad lo que se busca es información y conocimiento profundo -información y conocimiento usualmente más profundo que el buscado en encuestas, entrevistas informales o grupos focales, por ejemplo (JOHNSON, 2002)-, dado a que con cada pregunta de la entrevista a profundidad se busca obtener información específica y puntualizada que apunte a dar respuesta a alguno de los objetivos de información que tiene estructurados la entrevista. Por tal motivo, se interrogó a algunos docentes pertenecientes a la Facultad de Ciencias Económicas y Administrativas de la Corporación Universitaria Minuto de Dios, seccional Bello, con o sin experiencia en investigación, con el fin de evaluar los siguientes aspectos:

1) Conocimiento

2) Promoción

3) Percepción

4) Expectativa

5) Motivación

La evaluación de dichos criterios se implementa en docentes y se definen de acuerdo a las necesidades de estudio y a lo evidenciado en la literatura académica. La primera categoría Conocimiento, hace referencia al grado de familiaridad y entendimiento de los docentes con el sistema de investigación dentro Uniminuto, y que está enlazado a la siguiente categoría que es la Promoción, la cual, hace énfasis en la capacidad que tiene la institución y los docentes de incentivar y fomentar la investigación en los estudiantes durante su ciclo de formación académica. La literatura reporta que hay fallas recurrentes en la transmisión de 
la ciencia hacia los estudiantes, en la formación y en la comunicación de la actividad científica; lo cual tiende a disminuir notoriamente la participación de los estudiantes en experiencias investigativas (ROJAS, 2011). La tercera categoría es la Percepción, entendida como la imagen que tienen los docentes frente a los campos y líneas de investigación que desarrolla la institución, a la actividad investigativa, y al sistema de investigación; teniendo en cuenta que la participación de los estudiantes en experiencias de investigación se van a ver influenciadas por las percepciones que ellos tengan de estas, y que pueden servir como un motor de desarrollo o como un impedimento para que se lleve a cabo (ADEDOKUN; BURGESS, 2011).

La cuarta categoría a evaluar es la Expectativa, relacionada con los resultados que esperan obtener docentes y lo que los docentes creen que serán los resultados que esperan obtener los estudiantes a través de la participación en experiencias de tipo investigativas, como satisfacer intereses personales enlazados a la posibilidad de publicar, condiciones de desarrollo de una práctica investigativa, y desarrollo de las mismas actividades relacionadas con la investigación. Esta categoría se encuentra conectada con la quinta, que hace referencia a la Motivación, a través de la cual se busca indagar sobre los factores que llevan a los docentes a participar de los procesos investigativos que ofrece la institución y a lo que ellos creen que han de ser las motivaciones que llevan a los estudiantes a participar de los procesos investigativos. Finalmente, se desea contrastar los resultados obtenidos en la presente investigación con resultados de estudios anteriores; en los cuales se plantea que esas expectativas y percepciones están relacionadas con las promociones profesionales, el prestigio, la financiación (ÅKERLIND, 2008), la experiencia, el apoyo social (WOODCOCK et al., 2012), crecimiento personal, aprendizaje y remuneración económica (OSAMOR; KASS, 2012).

Algunas de las preguntas aplicadas a los docentes se muestran a continuación, referenciadas al objetivo de información que buscaba satisfacer,

\section{Cuadro 1}

\begin{tabular}{|c|c|c|}
\hline Aspecto & Preguntas & Comentarios \\
\hline Conocimiento & $\begin{array}{l}\text { 1. ¿Conoce las líneas de investigación institucionales? Mencione por } \\
\text { favor algunas de ellas. }\end{array}$ & $\begin{array}{l}\text { Se le pidió al docente que mencionara las líneas de investigación } \\
\text { para corroborar que efectivamente las conocía. }\end{array}$ \\
\hline \multirow{2}{*}{ Promoción } & $\begin{array}{l}\text { 1. ¿A través de qué mecanismos promueven la investigación como } \\
\text { eje articulador del proceso formativo en los estudiantes? }\end{array}$ & \multirow{2}{*}{$\begin{array}{l}\text { Eran tanto preguntas de lo que hacen los docentes para promover } \\
\text { la investigación en las aulas de clase, como lo que hace la } \\
\text { institución }\end{array}$} \\
\hline & $\begin{array}{l}\text { 2. ¿Considera efectiva las actividades de divulgación y difusión de las } \\
\text { actividades investigativas? }\end{array}$ & \\
\hline \multirow{2}{*}{ Percepción } & $\begin{array}{l}\text { 1. ¿Qué piensa sobre los campos de investigación que tiene } \\
\text { Uniminuto, desarrollo humano y desarrollo social sostenible? }\end{array}$ & \\
\hline & $\begin{array}{l}\text { 2. ¿Le parecen acordes las líneas de investigación establecidas desde el } \\
\text { centro de investigación de Uniminuto con los objetivos de FACEA? }\end{array}$ & \\
\hline \multirow{2}{*}{ Expectativa } & $\begin{array}{l}\text { 1. ¿Es aplicable para usted el desarrollo de investigación en estos } \\
\text { campos de investigación? }\end{array}$ & \multirow{2}{*}{$\begin{array}{l}\text { Aunque las preguntas de expectativa se hicieron todas en forma } \\
\text { de preguntar la percepción, con estas preguntas se podía inferir si } \\
\text { para el docente era importante ver sus investigaciones aplicadas } \\
\text { o publicadas o socializadas con la comunidad académica, etc. Y } \\
\text { por eso hacen parte del grupo de expectativas. }\end{array}$} \\
\hline & $\begin{array}{l}\text { 2. ¿Considera eficiente la gestión de la Dirección de Investigación } \\
\text { referente al proceso de publicación de libros basados en investigación? }\end{array}$ & \\
\hline \multirow{3}{*}{ Motivación } & $\begin{array}{l}\text { 1. ¿Cuáles han sido sus motivaciones para participar en el Grupo } \\
\text { de Investigación de la FCHE o desarrollar proyectos investigativos? }\end{array}$ & \multirow{3}{*}{$\begin{array}{l}\text { Se buscaban aspectos muy puntuales en la motivación, como } \\
\text { remuneración económica, reconocimientos en la comunidad } \\
\text { académica, generación de nuevos contactos, etc. Para poder } \\
\text { contrastar estos con los autores consultados } \\
\end{array}$} \\
\hline & $\begin{array}{l}\text { 2.¿Considera la remuneración económica una motivación para } \\
\text { participar dentro de proyectos investigativos en la Uniminuto? }\end{array}$ & \\
\hline & $\begin{array}{l}\text { 3. ¿Considera la interacción con comunidades investigativas y la } \\
\text { experiencia que se gana con esto, como una motivación importante } \\
\text { para participar en proyectos investigativos? }\end{array}$ & \\
\hline
\end{tabular}

Fuente: Elaboración propia para este estudio. 


\section{Análisis de resultados}

El análisis de resultados se realizará con base en las cinco categorías anteriormente descritas. Además, se espera contrastar los resultados encontrados en esta investigación con lo que se ha venido estudiando con respecto al tema y que se encuentra plasmado en la literatura académica.

En la primera categoría se evaluarán cuatro subcriterios: conocimiento sobre los campos de investigación, qué es un semillero de investigación, familiaridad con la oferta de semilleros de la Facultad de Ciencias Administrativas y Económicas de Uniminuto y conocimiento sobre la opción de práctica investigativa.

Dentro del estado del arte, Barrios define que la línea de investigación es considerada como:

[...] el eje ordenador de la actividad de investigación que posee una base racional y que permite la integración y continuidad de los esfuerzos de una o más personas, equipos, instituciones comprometidas en el desarrollo del conocimiento en un ámbito específico. (1990, p. 5).

La Facultad de Ciencias Administrativas y Económicas de Uniminuto ha definido campos de investigación institucional y para llevar a cabo la dinámica propiamente de las líneas de investigación, a partir del sistema se han generado semilleros de investigación como espacios de integración entre estudiantes y docentes.

En el mapa 1, se definen factores críticos sobre la categoría hallados durante el desarrollo de la investigación, así para la categoría de conocimiento las subcategorías son el conocimiento sobre el sistema en general y los esquemas de divulgación, planteando para cada uno, conforme a los resultados encontrados, las respuestas predominantes. Durante las entrevistas a docentes se evidenció que la mayoría de docentes conoce los campos, líneas y sub líneas, aunque algunos de ellos presentan confusiones en esos conceptos y en su diferenciación, tal como se muestra en el mapa 1 . Del mismo modo se percibe cómo ese conocimiento está enmarcado principalmente en su área o programa curricular; es decir, el profesorado conoce el campo, líneas y sub líneas de investigación, pero principalmente de su área y reconoce que los otros miembros de la facultad hacen igualmente sus esfuerzos, pero desconocen en qué. Además, según la muestra de entrevista analizada, se identifica que posiblemente hay ausencia de una capacitación informativa sobre estos temas al ingresar a la institución, ya que la mayoría de los profesores conocen lo que han hecho y específicamente en las líneas que han participado, ya sea en su conformación o en su proceso de desarrollo; pero no conocen lo que se ha trabajado tiempo atrás, ni lo que se ha construido desde antes, lo que quiere decir igualmente, que los docentes cuando llegan a la organización inician desde cero y no continúan con los procesos que ya se han venido desarrollando. Esto se identificó debido a situaciones como esta: se le preguntó a uno de los docentes si conocía las líneas de investigación. La respuesta fue "Tenemos la, la, (titubea) la línea de la facultad, que trabaja todo lo que es administrativo, pues el nombre exacto no me lo sé [...]" (Entrevista ${ }^{\circ} 1$, Bello, abril, 19 2013). Con lo anterior se percibe inmediatamente como el docente realmente no conoce mucho sobre las líneas de investigación. En una pregunta posterior el profesor dice "[...] desde que yo llegué, ya las líneas de investigación estaban de lo que sí hice parte fue de las sub líneas de investigación, que ya pertenecen pues, a mi programa” (Entrevista $n^{\circ} 1$, Bello, abril, 19 2013). Respuestas similares se identifican en otras entrevistas realizadas. En algunos casos específicos, los docentes manifiestan conocimiento sobre las líneas de investigación, sin recordar específicamente los nombres de éstas, pero se evidencia que son conocedores de la orientación de esos enfoques de investigación y de la existencia de diferentes líneas dentro de la misma institución, reconociendo que desde cada área de estudio que tiene definida una línea se busca lograr soluciones relacionadas con el 
entorno, cumpliendo con el propósito de los campos de investigación de la institución. En relación a los semilleros el centro de investigación en sinergia con las facultades y las coordinaciones de programas académicos generan una oferta de espacios para la participación de los estudiantes, estos espacios son conocidos dentro de la institución como sub líneas de investigación, y son altamente valorados por los docentes, reconociendo que a través de este tipo de prácticas se logra un gran enriquecimiento académico mediante la socialización de conocimientos, dentro y fuera de la institución, así mismo reconocen el esfuerzo que ha hecho la institución por avanzar y consolidar equipos de investigación con docentes aptos y en apoyar el desarrollo de competencias y formación de docentes que tienen el potencial para apoyar procesos de investigación.

Mapa 1- Conocimiento de los procesos investigativos

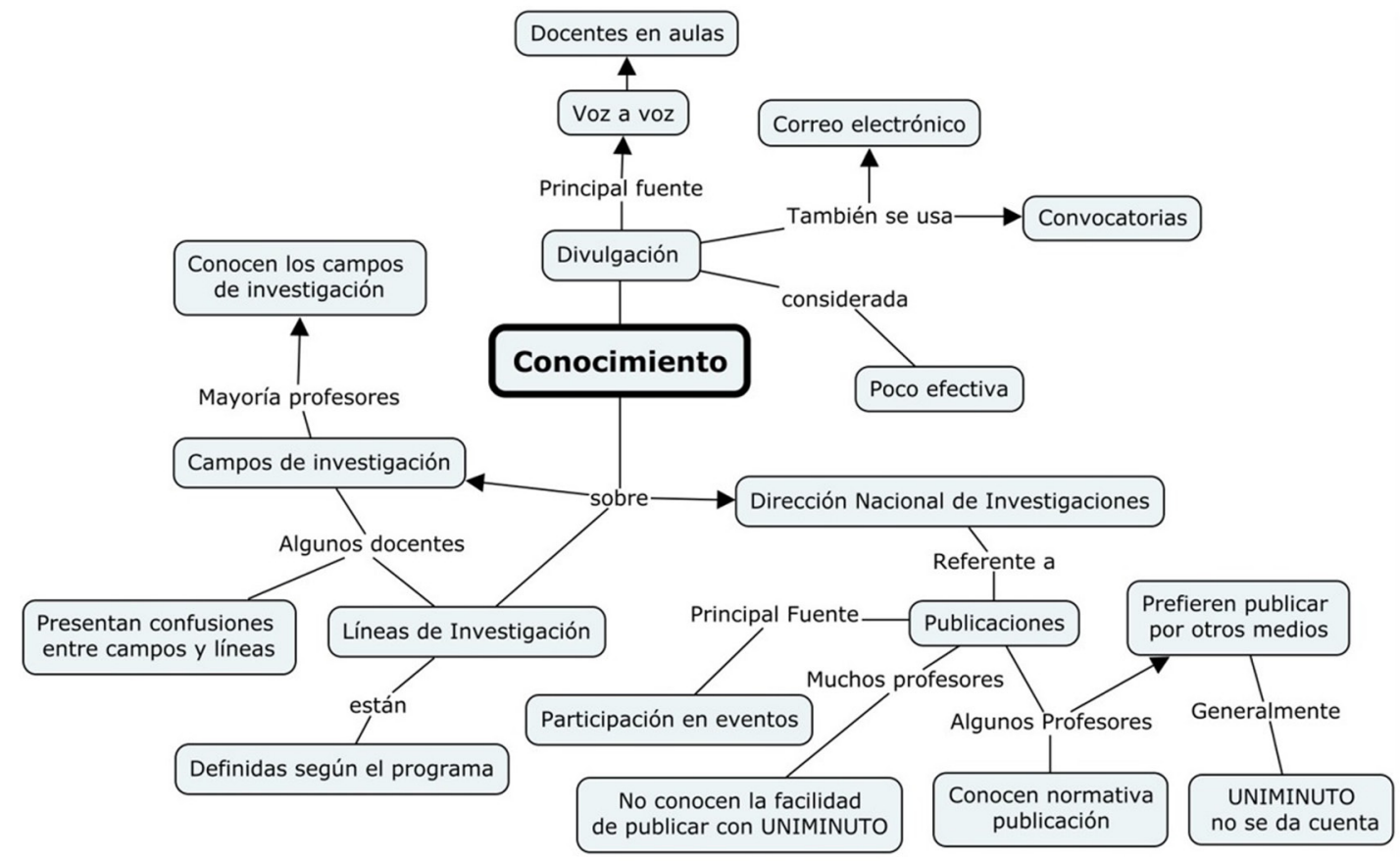

Fuente: Elaboración propia a partir de la aplicación del instrumento.

Respecto a que tanto los profesores están enterados de la publicación para dar a conocer sus investigaciones a través de Uniminuto, se encontró que si bien muchos docentes aún no están informados de esa posibilidad que les brinda la universidad, algunos otros prefieren publicar por otros medios. Es conveniente resaltar que para los profesores

La publicación en una revista reconocida por el área es la manera más aceptada para registrar la originalidad del trabajo y para confirmar que los resultados son suficientemente fiables para superar el escepticismo de la comunidad científica. (SCHWARZ; BORGES, 2012, p. 79).

Del mismo modo las oportunidades de un autor para aumentar su visibilidad, son entonces dependientes de su mayor exposición a la comunidad mundial de pares y eso tiene más probabilidad de ocurrir cuando una publicación de trabajos de investigación se da 
en periódicos internacionales, principalmente aquellos indexados en bases de datos especializadas e internacionales, de amplia divulgación y prestigio (MUELLER, 2004 apud ZIMBA; MUELLER, 2004ํ).

De esta forma puede inferirse que una de las razones de peso por la que los maestros de la Universidad objeto de estudio prefieren publicar en otros canales de difusión del conocimiento puede ser la calificación y prestigio que tienen los medios de publicación de la Uniminuto respecto a la comunidad académica. Del mismo modo, los líderes de grupo y los jefes de dependencia de Uniminuto no se enteran de las publicaciones que hacen sus docentes en otros medios, lo que puede ser un factor desmotivante para la producción científica de estos docentes. Sin embargo, algunos docentes conocen los lineamientos a nivel general para la publicación de artículos desde la institución y adicionalmente en algunos casos reconocen la disposición de los equipos de apoyo sobre dichos procesos, pero enfatizan en la falta de divulgación de esta oportunidad.

Con relación a la promoción y capacidad de la institución por incentivar y fomentar la investigación el Sistema Universitario Minuto de Dios ha definido como campos de investigación institucional el desarrollo humano y el desarrollo social sostenible. Dentro de Uniminuto la investigación, fuente del saber, generadora y soporte del ejercicio docente, tiene como finalidad la generación y comprobación de conocimientos orientados al desarrollo de la ciencia, de los saberes, de la técnica, la producción y adaptación de tecnología para la búsqueda de soluciones a los problemas de la región y del país, con miras a la transformación e impacto social. Es por esto que las actividades de difusión y divulgación juegan un papel fundamental en

1- Traducción nuestra. la transmisión de los procesos de investigación que se están llevando a cabo desde el centro de investigación y de sus propias funciones.

Los profesores también reconocen que han sido la principal fuente de divulgación y comunicación de la oferta investigativa y que ésta se ha basado en el voz a voz primordialmente y en el trato cercano con las personas. No sólo es el medio por el cual se enteran los estudiantes de los procesos investigativos a desarrollar, sino también la forma en cómo los docentes se actualizan y se dan cuenta de los nuevos avances en cuanto a temas de investigación de la institución. Herramientas más formales como el correo electrónico, la intranet, o convocatorias son utilizadas con menor frecuencia. Al igual que los estudiantes, los docentes consideran que el canal de comunicación es poco efectivo, así como lo expresa explícitamente uno de ellos "[...] la gente no sabe lo que estamos haciendo acá [...]" (Entrevista $n^{\circ} 5$, Bello, abril, 19 2013). En el mapa 2 se presentan las principales respuestas dadas por lo docentes con respecto a las actividades que deben ser fomentadas a partir de la promoción. Los docentes proporcionaron respuestas relacionadas con los esquemas actuales de divulgación, la cultura investigativa y su papel dentro de la institución como ente promotor de los procesos de investigación. Ellos recomiendan una página web donde los estudiantes puedan estar informados de las actividades que se realizan, tal y como lo expresa una docente de Uniminuto:

[...] todo lo que conozco es por otros profesores que me cuentan. También vienen algunos correos electrónicos, pero me parece que hay que organizar mejor esta parte, tener más información. Tenemos que tener algunos sitios de red donde tú puedas consultar en cualquier momento [...]. (Entrevista nº, Bello, abril, 19 2013). 
Mapa 2- Promoción de los procesos investigativos

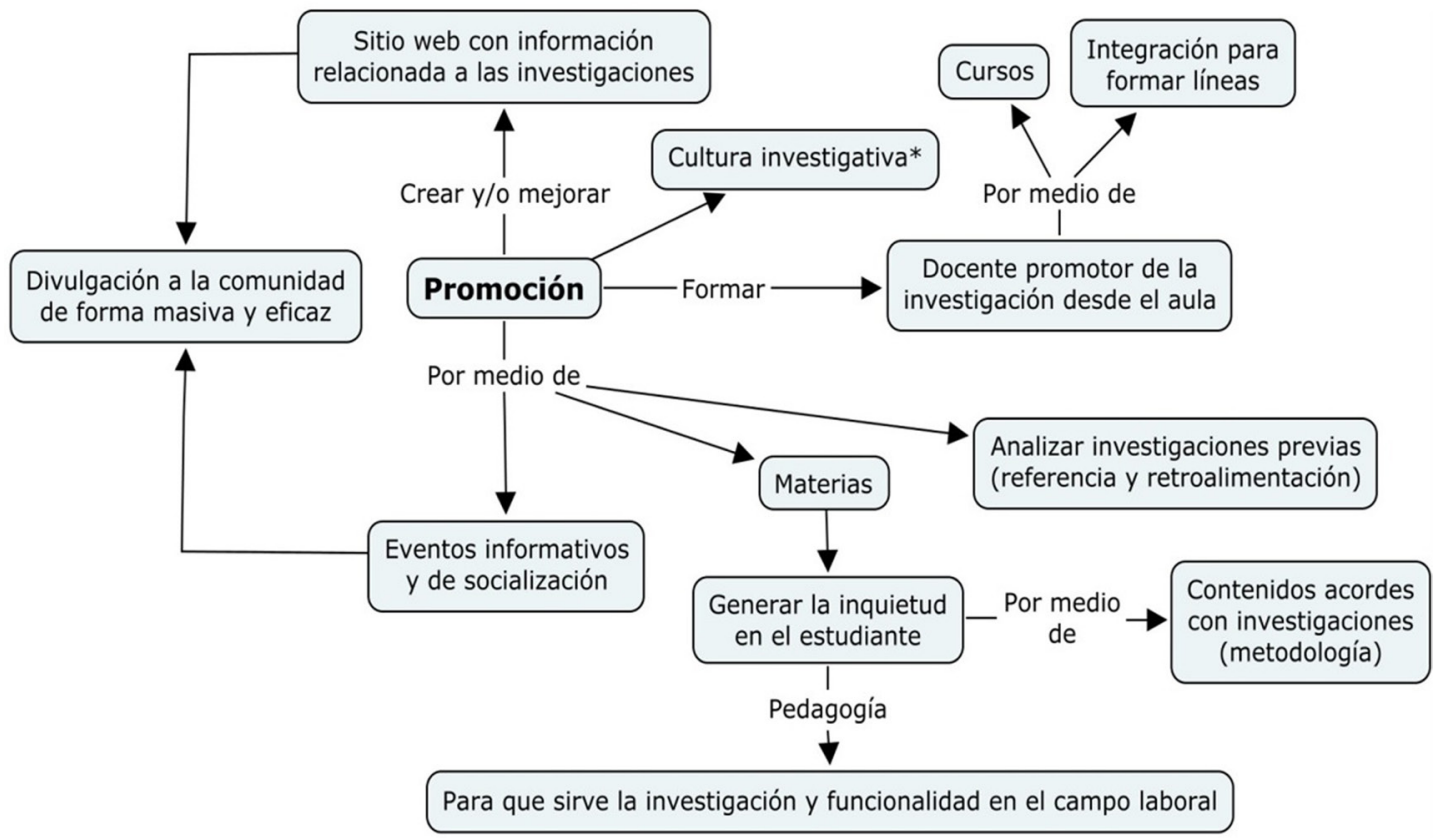

Fuente: Elaboración propia a partir de aplicación del instrumento.

Si bien el voz a voz ha sido el medio de comunicación por excelencia a través de los tiempos entre estudiantes y maestros, es importante ir a la vanguardia con las nuevas tecnologías y los medios de comunicación, generar una cultura dentro de la institución relacionada con la amplia oferta de medios de comunicación. En efecto, poner en práctica dentro de las instituciones los beneficios que el desarrollo tecnológico ha traído consigo es un movilizante de una comunicación más rápida y efectiva en el interior de las instituciones (DE AGUILERA; BATLLE; FERNÁNDEZ, 2012).

Los docentes manifiestan la necesidad de mostrarles a los estudiantes experiencias investigativas previas para que tomen esto como ejemplo y punto de partida, como lo dice una docente de Uniminuto: "la idea es promover que los estudiantes se motiven por la investigación. Muchas veces ellos necesitan ver como un referente en otras personas o en otros jóvenes que estén investigando [...]" (Entrevista $\mathrm{n}^{\circ} 2$, Bello, abril, 19 2013). Lo anterior, tal y como lo expresa la docente, podría ser un recurso muy adecuado para influenciar a los jóvenes a procesos investigativos.

Medina (2009) expresa que para retener, identificar y atraer investigadores de alta calidad es fundamental tener en cuenta aspectos como las relaciones sociales y la infraestructura percibida para la investigación. Este autor, en sus investigaciones, recalca que un factor de motivación para que las personas se dediquen a la investigación es ver que hay apoyo para esto; es decir, que se cuenta con buenos laboratorios, espacios, tecnología y recursos. Del mismo modo es una motivación pertenecer a un contexto investigador, ver espejos exitosos de sus semejantes en el campo de la investigación. Por ejemplo, González menciona que "los investigadores que más publican (productividad muy alta) son los que están más interesados en trabajar con colegas de alta competencia" (1979, p. 71), lo que refleja que ser investigador genera un cierto poder o estatus en la sociedad. En las entrevistas se evidencia que los docentes consideran un factor motivante la interacción con otras 
comunidades académicas y el intercambio de experiencias, resaltando la importancia de conocer lo que están desarrollando otros, pero también enfatizando como factor motivante el reconocimiento dentro de la comunidad académica, a partir del desarrollo de procesos de investigación que son puestos en común en eventos como semilleros, actividades de socialización de investigaciones, etc.

Algunos profesores de Uniminuto piensan que otro problema que se presenta es la falta de cultura en la institución, ya que los estudiantes confunden el concepto de investigación y no saben de forma correcta para qué sirve, como lo afirma una docente:

[...] es enseñarles a los estudiantes para qué sirve la investigación, porque normalmente lo ven como un ladrillo, como algo pesado, algo pues maluco. Entonces si no se le da una buena pedagogía a la forma en cómo se está enseñando la investigación, digamos que los resultados no van a ser tan buenos. (Entrevista n8, Bello, abril, 19 2013).

Los docentes consideran que es necesario romper con el imaginario de cómo ha sido vista la investigación, de que es apta solo para un público específico y de que hay carreras en las que no hay oportunidad de investigar, y para esto es necesario empezar a mostrar la investigación como un proceso flexible, que permite crecer académica y personalmente, que abre puertas a la participación en eventos académicos, a publicaciones y a potencializar las competencias de quienes participan de ellas. Indican que cambiar estos paradigmas no se hace a través de acciones de mercadeo masivo, sino mediante acciones concretas de docentes comprometidos, que incentiven la participación de los estudiantes en procesos de investigación con metodologías menos rígidas que despierten la curiosidad del estudiante. Se evidencia así la necesidad de formar docentes capacitados para promover la investigación desde el aula de clase con el fin de generar la inquietud en el estudiante y que esto lo lleve a participar en los procesos investigativos.

Referente a la promoción de la investigación que se hace en Uniminuto, ésta ha de incluir tanto a profesores, directivos y estudiantes, donde todos se apoyen y el esfuerzo sea mancomunado, desde la misma formación de líneas donde los profesores piden que sean más incluidos, hasta los temas de las clases, dado que es allí donde se debe motivar al estudiante con temas acordes. Para todo esto se debe tener un canal de comunicación donde la interacción y la información sea importante y útil para todos, e ir formando así, poco a poco, una cultura investigativa dentro de la institución.

En cuanto a la percepción de los docentes referente al tema de investigación, cabe resaltar que la participación en este tipo de experiencias está altamente influenciada por la imagen que tengan ellos de estos procesos, y que la participación en experiencias investigativas está relacionada con resultados académicos positivos para los estudiantes (ADEDOKUN; BURGESS, 2011). Es por esta razón que se hace pertinente conocer si los campos de investigación propuestos por el Sistema Universitario Minuto de Dios (educación, transformación social e innovación, desarrollo humano y comunicación, innovaciones sociales y productivas, gestión social, participación y desarrollo comunitario), son del agrado de la comunidad académica; por lo cual se hace relevante evaluar la imagen que tienen tanto estudiantes como docentes de estos campos y líneas de investigación en cuanto a su pertinencia, importancia, y aplicabilidad.

En el mapa 3 se identifican las principales revelaciones de las entrevistas relacionadas con cómo perciben los docentes diferentes procesos, desde el concepto de investigación, los otros docentes, procesos de socialización, estudiantes y los enfoques investigativos de la institución, se evidencia así que la mayoría de los profesores consideran que los campos de investigación son pertinentes y que están alineados con la misión y visión de la universidad, rescatándose de éstos un enfoque 
y concepción del profesional, diferente al resto de universidades, dado a que según los docentes en la institución vale tanto lo académico como lo humano, y estos campos están orientados precisamente a una visión diferente, a través de la cual se explora no solo la parte académica sino también una parte muy humanitaria y adicionalmente consideran que es un campo muy aplicable, especialmente en la realidad del país de la institución y que indudablemente pueden aportar a la transformación de la sociedad.

Mapa 3- Percepciones de los procesos investigativos

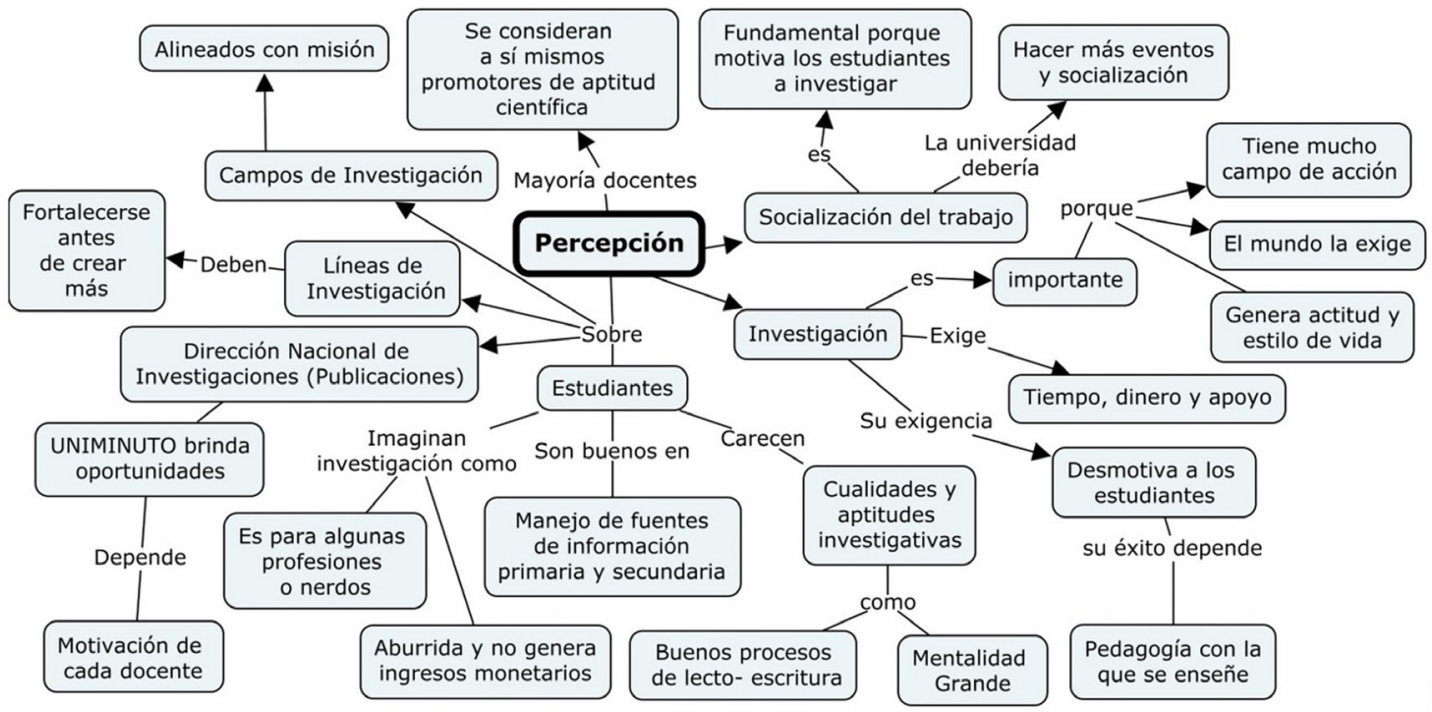

Fuente: Elaboración propia a partir de la aplicación de la herramienta.

Los profesores consideran que la formación de las líneas de investigación ha sido un proceso participativo, en el que se han visto involucrados. Pero del mismo modo también piensan que aún se está en ese camino de construcción y que es necesario tener visiones y planteamientos claros en las líneas que ya se tienen, antes de pensar en crear alguna otra.

Además, los docentes piensan que los estudiantes perciben la investigación como una tarea aburrida, que corresponde a personas intelectuales y de algunas carreras en especial y que esto de una u otra forma sesga los procesos investigativos, y la motivación que estos puedan sentir hacia ellos. Además, consideran que esta clase de creencias viene debido al entorno cultural que rodea a los estudiantes.

Ante esto Castillo, propone diez estrategias para la formación de investigadores, siendo una de estas la de desmitificar la investigación científica.

La investigación no es una disciplina individualista ni oculta, delimitada a unas pocas personas y escasas mentes privilegiadas, o 'genios' con vocación para ello, [...], la investigación se encuentra al alcance de quien se lo proponga. (CASTILLO, 2007, p. 22).

Por consiguiente, es un deber de la formación y del docente romper este paradigma en los estudiantes, para invitarlos al mundo de la investigación.

Algunos profesores consideran que los estudiantes perciben la investigación como una actividad que no genera ingresos monetarios, mientras que el trabajo en una empresa sí lo 
hace. Sin lugar a duda, los docentes por su parte consideran que la investigación trae consigo una nueva forma de vida, y que es totalmente útil y aplicable a la vida práctica. Sólo uno de los docentes entrevistados consideró que las líneas de investigación no son realmente aplicables a los estudiantes, dado a que estos trabajan y eso hace esta misión compleja.

Autores como Medina (2009) y González (1979) informan como resultado de sus investigaciones que un factor para que las personas se motiven a investigar son los incentivos económicos. Desde esta perspectiva sería un deber de los educadores romper el esquema de los estudiantes y demostrarles que es posible recibir beneficios económicos por investigar, aunque no parezcan tan plausibles y de fácil acceso. "Los incentivos económicos existen, el problema es que no se ofrecen de forma uniforme" (MEDINA, 2009, p. 42). González (1979) llegó a la conclusión que el 18,8\% de los investigadores que participaron en su estudio confesaron que se vincularon al mundo de la investigación porque era un método de poder ganar dinero. En los resultados de su investigación se plasma que aunque el dinero no es la razón principal por la que las personas dedican su vida a la investigación y que aunque en general los investigadores perciben ingresos moderados por su trabajo, el dinero sí hace parte de los factores de decisión para dedicarse a la investigación como profesión y no a otras actividades. En las entrevistas se evidencia como factor motivante la retribución económica reconociendo la investigación como un proceso que requiere dedicación y tiempo para lograr resultados.

Teniendo en cuenta el contexto de Uniminuto y viendo que la mayoría de los estudiantes se encuentran inmersos en el mundo laboral empresarial, tal vez es pretencioso esperar que estos sacrifiquen su fuente de ingresos para dedicarse a la investigación no remunerada, pero si los estudiantes ven una posibilidad de obtener dinero con ésta, quizás éste sí sea un factor determinante para decidir convertirse en investigadores.
Del mismo modo, es importante analizar que el tiempo que los estudiantes de la Uniminuto dedican tanto al trabajo, que es mínimo 30 horas semanales y máximo 48, según el artículo 161 del Código Sustantivo del Trabajo (COLOMBIA, 1950), como a su formación universitaria, permiten inferir que la disponibilidad horaria del alumnado para los ratos de ocio no es muy alta; por tanto, el motivo principal por el que los estudiantes prefieren dedicar sus ratos libres al trabajo investigativo, que a otras cosas recreativas (deporte, cine, fiestas, etc.) es el gusto propio por el arte. Lo que habría que cuestionarse ahora es cuántos estudiantes de la institución sienten tanto gusto por la investigación, para ser capaces de sacrificar sus espacios de recreación por ésta.

Los docentes consideraron la investigación como una posibilidad para su formación profesional, ya que en su mayoría, a la pregunta de las motivaciones para ingresar al mundo de la investigación, siempre mencionaron algún aspecto con la educación académica formal, de la cual aún están en proceso de formación. Argumentos como los estudios de maestría y doctorado, estuvieron presentes como motivación de los procesos investigativos.

Lo anterior es coherente con lo evidenciado en un estudio realizado por Osamor y Kass (2012), en el cual se obtuvo que los estudiantes consideran como factores motivacionales para participar en grupos de investigación, aspectos como el crecimiento personal, aprendizaje y beneficio económico, todos intereses muy personales. Así mismo González (1979) dice que el 9,8\% de los investigadores que participaron en su estudio, se motivaron a convertirse en investigadores para ascender de escala académica con expresiones como "en principio, para hacer la maestría o el doctorado" (p. 7). En el mismo orden de ideas también agrega que el 11,1\% se motivó porque "era importante para dedicarse a la docencia” (p. 7).

En general, la mayoría de los docentes pone en primer lugar el crecimiento personal 
como motivación principal, pero no dejan de lado los aspectos como remuneración económica y tiempo. Esta idea se complementa con lo expuesto por Medina (2009) y González (1979), quienes encontraron en sus estudios que la principal razón porque los investigadores decían haberse motivado a dedicarse a la investigación era el gusto por el oficio o porque sintieron que ésta era su vocación en la vida. Para el desarrollo de una investigación se necesitan estos dos elementos (tiempo y financiación), razones por las cuales algunos de los profesores no se han involucrado en grupos de investigación, aunque tal vez éste haya sido su deseo. También vale la pena decir que todos los docentes entrevistados consideran que la remuneración económica sería una buena motivación para el desarrollo de procesos investigativos, sin embargo, bajo el esquema de funcionamiento actual de la institución, en el cual los docentes no reciben una remuneración monetaria adicional por participar en procesos de investigación o tener publicaciones indexadas como ocurre en otras instituciones del mismo nivel, se evidencia que tienen como motivación principal su desarrollo académico y personal, incluso en casos en los que algunos no tienen una formación de maestría y doctorado.

Del mapa 3 se ve como la mayoría de docentes se consideran a sí mismos como promotores de aptitud científica y reconocen que los estudiantes son buenos en el manejo de fuentes de información primaria y secundaria; por lo que básicamente la promoción de aptitud científica que los docentes han hecho sobre los estudiantes se ha centrado en construir las buenas habilidades para el dominio de estas fuentes. A lo largo de dicho mapa y de las entrevistas se encuentra que los maestros no se perciben a sí mismos como parte del problema. Siempre toman una posición neutral y proponen soluciones que corresponden a la Uniminuto (hacer más eventos de socialización, crear una página web, hacer difusión masiva, etc.).

En el mapa 4, se enuncian las expectativas que tienen los docentes para la investigación en el plantel con respecto a los esquemas actuales y a la percepción del futuro de la investigación dentro de la institución. Casi en todos los casos, las expectativas recaen sobre la institución Uniminuto como ente que debe propiciar el cambio.

\section{Mapa 4- Expectativas de los procesos investigativos}

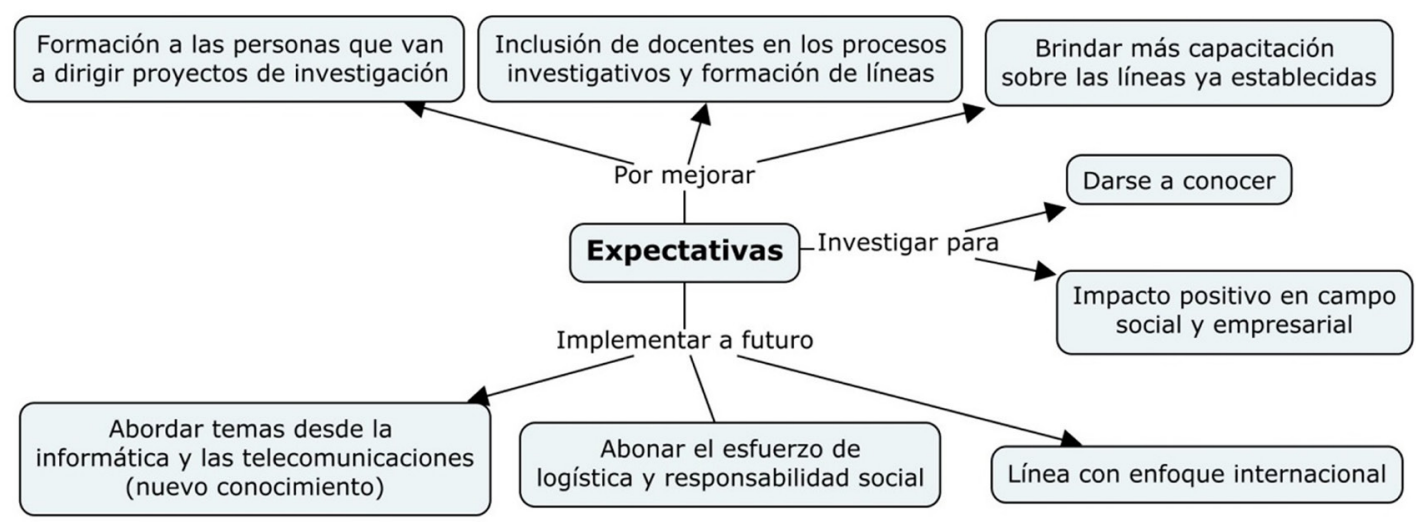

Fuente: Elaboración propia a partir de aplicación del instrumento.

En cuanto a las expectativas, los docentes piensan muy similar en ciertos aspectos que se deben mejorar. Una de las cosas por mejorar es la formación de los docentes como afirma el coordinador del Centro de Emprendimiento: "Creo que la universidad, que apenas está en 
ese proceso, debe tomar conciencia de formar a las personas que vamos a estar detrás de proyectos de investigación [...]" (Entrevista $\mathrm{n}^{\circ} 1$, Bello, abril, 19 2013). Esto con el fin de tener profesores con la capacidad suficiente de transmitir los conceptos y la metodología de la investigación a los estudiantes.

Cuando se les preguntó a los docentes sobre qué líneas pensaban pertinentes implementar a futuro, la gran mayoría dijo que no era necesario esto, que era más importante profundizar en las líneas ya establecidas, impulsarlas, invertir en ellas, darlas a conocer en el medio, no solo a nivel nacional sino internacional y abrir las puertas de la institución al intercambio de conocimientos, como lo reafirma un docente: "Yo creo que la universidad tiene que empezar a hacer investigaciones para darse a conocer. Investigaciones que impacten social, empresarialmente [...]" (Entrevista $n^{\circ} 5$, Bello, abril, 19 2013). Aprovechando que la universidad se enfoca en diferentes áreas.

También fue posible encontrar nuevas propuestas. Un profesor considera que: “[...] deberíamos abordar temas específicos que generen un nuevo conocimiento desde lo que es la informática y el teleco, las telecomunicaciones, o sea el desarrollo de nuevas tecnologías o nuevos procesos también" (Entrevista $n^{\circ} 7$, Bello, abril, 19 2013). Se evidencia así la necesidad de empezar a abordar e investigar temas de más actualidad, que puedan llevar a motivar no sólo a docentes sino también a los mismos estudiantes. Es así como también otra profesora afırma: "Yo pienso que hay que tener más parte internacional” (Entrevista $n^{\circ} 3$, Bello, abril, 19 2013). Se infiere que esta cualidad podría ser una debilidad de los procesos investigativos actuales, y que si se corrige, se transformaría en un gran atractivo. Es necesario entonces ampliar el pensamiento y el horizonte con el fin de recibir una retroalimentación.

En general, las expectativas más recurrentes en los entrevistados son, por una parte, la de ampliar la proyección de los procesos investigativos al exterior. Esto con el objetivo de darse a conocer, conocer nuevos procesos que retroalimenten los actuales y generar un impacto positivo en lo social y empresarial; y por la otra, la formación de los docentes, los cuales quieren ser promotores de investigación en sus cursos, contando con las herramientas necesarias para esto y así emprender procesos investigativos con sus alumnos.

\section{Conclusiones}

Es posible abordar investigaciones de este tipo, -en donde se quiere tratar un problema o una situación general, en este caso, relacionados con las percepciones de los docentes en cuanto a los procesos de investigación llevados a cabo por sus respectivas universidades- en una primera instancia desde el análisis de estudios de casos, como en el presente estudio en donde se aplicaron encuestas a docentes de la Facultad de Ciencias Económicas y Administrativas, con el objetivo de conocer sus percepciones en cuanto a los procesos investigativos; puesto que no solo se logra validar algunos puntos propuestos por diferentes estudios y autores en la literatura, sino también considerar algunos factores clave para la aplicación y extensión en otros casos.

La investigación, en términos generales, permite concluir que la situación más importante a evaluar, referente a los procesos investigativos desarrollados en la Facultad de Ciencias Económicas y Administrativas de Uniminuto, es el papel que está jugando la actividad de divulgación del sistema de investigación dentro de la institución, ya que con las entrevistas fue posible observar que existe un desconocimiento general de los docentes acerca del enfoque de investigación de la institución y de las oportunidades de las cuales pueden ser partícipes, como por ejemplo las publicaciones que pueden hacer. También se encontró que el profesorado se encuentra familiarizado con la investigación de sus áreas y que desconocen en cierta medida lo que ocurre con otros campos dentro de la misma institución. Dado esto, es importante desarrollar actividades 
de divulgación y promoción de los procesos investigativos dentro de la institución, con el fin de incentivar la participación más activa de los docentes y lograr que los estudiantes, pese a las condiciones socioeconómicas, se unan a estos procesos y conozcan los beneficios que éstos pueden proporcionarles.

Existe un reto especial relacionado con romper con imaginarios y paradigmas que se han perpetuado en algunas áreas de estudio $\mathrm{y}$ se ha mostrado la investigación como un proceso aburrido, rígido y solo apto para ciertas carreras profesionales. Sin embargo, pese a la poca participación de los estudiantes en procesos de investigación, como lo son los semilleros de investigación dentro de Uniminuto, se encontró que ellos asocian la investigación a una actividad innovadora, enriquecedora, que capacita y aporta desarrollo, lo cual puede indicar un buen potencial en la mente de los estudiantes para lograr conectar las aulas de clases con los procesos de investigación y de este modo fomentar no sólo una cultura investigativa, sino también orientar la investigación a los intereses de los estudiantes.

Se observó que el tiempo es una variable determinante en la participación de los estudiantes en procesos de investigación y esto puede deberse a que varios de los alumnos son personas que trabajan y estudian de una manera paralela. El tiempo es el factor más importante para ellos a la hora de emprender una investigación y consideran que no se motivan a realizar una práctica investigativa por el tiempo que ésta consume. La institución en este sentido, debe reestructurar los esquemas de participación de los estudiantes, a través de prácticas que les permita mantener un equilibrio entre su vida laboral y académica, pero con niveles de exigencia con los cuales se logren resultados de calidad. Esto puede llevarse a cabo desde el desarrollo mismo de las materias durante los semestres académicos, en los que los estudiantes desarrollen sus propios procesos de investigación orientados a las líneas de investigación de la institución y que dichas experiencias a través de los docentes, les dé a conocer los beneficios de participar en investigación.

Aunque la mayoría de los docentes manifestaron aspectos relacionados con el crecimiento personal y con la vocación como motivadores hacia la investigación, es importante considerar los aspectos económicos, ya que todos los docentes entrevistados consideraron que la remuneración económica sería una buena motivación para el desarrollo de procesos investigativos, al ser procesos de dedicación y tiempo y que finalmente requiere de exigencia para lograr resultados aptos. De igual forma, una motivación para que los estudiantes se sumerjan en procesos de investigación es demostrarles que es posible recibir beneficios económicos por investigar, aunque no parezcan tan plausibles y de fácil acceso.

Con la investigación realizada se hace evidente la falta de capacitación a los docentes, en cuanto a los procesos de investigación y al desarrollo de los proyectos orientados hacia este fin, con el objetivo de poder transmitir los conceptos y la metodología de la investigación a los estudiantes. La institución, desde el centro de investigación y las coordinaciones académicas de cada programa, debe asumir el compromiso de apoyar a los docentes en su formación, buscar apoyos externos y crear sinergias con instituciones y diferentes comunidades académicas para generar espacios de intercambio de experiencias, conocimientos y aprendizaje, que aporten a los mismos docentes y a los estudiantes.

En Uniminuto hay un potencial muy bueno en investigación. Tanto profesores como estudiantes quieren participar en procesos investigativos. El problema radica, tal vez, en la falta de motivación brindada a la comunidad en general y en la falta de apropiada promoción. Es así como muchas personas no saben qué campos y líneas son las trabajadas en Uniminuto y no encuentran una verdadera ganancia en la participación en este tipo de procesos. Si bien el voz a voz ha sido el medio de comunicación por excelencia para realizar la promoción de la actividad investigadora, es 
importante ir a la vanguardia con las nuevas tecnologías y los medios de comunicación, para que la información esté disponible en todo momento. Es necesario que la universidad implemente un modelo de comunicación más agresivo en línea con los medios actuales, no solo para promover los temas relacionados con investigación, sino todo tipo de información, y que estos se conviertan los canales a través de los cuales se logre una mayor receptividad.

Se encuentra dentro de la institución un problema relacionado con la no satisfacción de las expectativas que tienen los profesores respecto a los procesos investigativos, por lo que es necesario una reestructuración y una reorientación de algunas líneas, con el objetivo de fortalecer los procesos en el interior de la universidad y crear lazos de cooperativismo con otras instituciones, tanto a nivel nacional como internacional, generando así alianzas con la comunidad académica, apoyo de las entidades gubernamentales y una mayor inversión en en la generación de espacios de conocimiento e investigación.

\section{Referencias}

ADEDOKUN, Omolola; BURGESS, Wilella. Uncovering students' preconceptions of undergraduate research experiences. Journal of STEM Education, Auburn, v. 12, n. 5, p. 12-22, 2011.

AGUIRRE, Julia. La percepción que tienen los colombianos sobre la ciencia y la tecnología. Bogotá: Departamento Administrativo de Ciencia Tecnología e Innovación, 2005. p. 1-239. Disponible en: <http://hdl.handle.net/11146/263>. Acceso en: 15 feb. 2015.

ÅKERLIND, Gerlese. An academic perspective on research and being a researcher: an integration of the literature. Studies in Higher Education, Oxford, v. 33, n. 1, p. 17-31, 2008.

ARAI Ken-Ichi et al. The future of research universities. Is the model of research-intensive universities still valid at the beginning of the twenty-first century? EMBO Reports, London, v. 8, n. 9, p. 804-810, 2007.

ASSOCIATION OF AMERICAN UNIVERSITIES. University research: understanding Its role. Washington: NW, 2011. Disponible en: <http://files.eric.ed.gov/fulltext/ED517265.pdf>. Acceso en: 09 feb. 2015.

ASTIN, Alexander; ASTIN, Helen. Undergraduate science education: the impact of different college environments on the educational pipeline in the sciences. Los Angeles: Eric, 1992. Final Report. Disponible en: <http://eric.ed.gov/?id=ED362404>. Acceso en: 09 feb. 2015.

BARRIOS, Marcos. Criterios y estrategias para la definición de líneas de investigación y prioridades para su desarrollo en Plan Integrado de Investigación. Taller celebrado bajo los auspicios del vicerrectorado de investigación y postgrado. Caracas: Universidad Pedagógica Experimental Libertador, 1990. p. 1-14. Mimeografiado.

CALLEJO, Javier. Observación, entrevista y grupo de discusión: el silencio de tres prácticas de investigación. Revista Española de Salud Pública, Madrid, v. 76, n. 5, p. 409-422, 2002.

CASTILLO, Mauricio. Identificación de estrategias para la formación de investigadores desde la escuela. Studiositas, Bogotá, v. 2, n. 2, p. 20-29, 2007.

COLOMBIA. Decreto Ley 2663, del 05 de agosto de 1950. Código Sustantivo del Trabajo, Bogotá, Capítulo II, artículo 161. Disponible en: <www.alcaldiabogota.gov.co/sisjur/normas/Norma1.jsp?i=33104>. Acceso en: 01 mar. 2015.

COLOMBIA. Ministerio de Educación. ¿Qué tanto promueven la investigación las universidades en Colombia? Centro Virtual de Noticias - CVN. Bogotá: ME, 2005. Disponible en: <www.mineducacion.gov.co/cvn>. Acceso en: 22 feb. 2015.

DAZA-CAICEDO, Sandra et al. Percepciones de las ciencias y las tecnologías en Colombia: resultados de la III Encuesta Nacional de Percepción Pública de la Ciencia y la Tecnología. Bogotá: Observatorio Colombiano de Ciencia y Tecnología, 2014. p. 01-388. Disponible en: <http://percepcionescyt.ocyt.org.co/\#>. Acceso en: 15 feb. 2015. 
DE AGUILERA, Miguel; BATLLE, Pedro Farias; FERNÁNDEZ, Antonio Baraybar. La comunicación universitaria. Modelos, tendencias y herramientas para una nueva relación con sus públicos. Revista ICON014, Madrid, v. 8, n. 2, p. 90-124, 2012.

GONZÁLEZ, Pedro. Motivación y productividad en la investigación científica española. Reis, Madrid, v. 7, n. 79, p. 63-75, 1979. (Está correto?)

GONZÁLEZ, Santiago. Formación investigativa para la educación superior desde una perspectiva pedagógica. Revista Científica, Bogotá, v. 14, n. 1, p. 72-78, 2011.

HATHAWAY, Russel; NAGDA, Biren; GREGERMAN, Sandra. The relationship of undergraduate research participation to graduate and professional education pursuit: an empirical study. Journal of College Student Development, Baltimore, v. 43, n. 5, p. 614-631, 2002.

HOFSTEIN, Avi; LUNETTA, Vicent. The laboratory in science education: Foundations for the twenty-first century. Science Education, E.E.U.U., v. 88, n. 1, p. 28-54, 2004.

IRVINE, John; MARTIN, Ben. Foresight in science: picking the winners. London: Pinter, 1984.

JOHNSON, John. In-depth interviewing. In: GUBRIUM, Jaber; HOLSTEIN, James (Ed.). Handbook of interview research: context and method. Thousand Oaks: Sage, 2002. p. 103-119.

LEGARD, Robin; KEEGAN, Jill; WARD, Kit. In-depth interviews. In: RITCHIE, Jane; LEWIS, Jane (Ed.). Qualitative research practice: a guide for social science students and researchers. London: Sage, 2003. p. 138-169.

MEDINA, Zulma. Estudio sobre las destrezas y habilidades de un investigador en la nueva era del conocimiento. Puerto Rico: Consejo de Educación Superior de Puerto Rico, 2009.

MIRANDA, Rommel; DAMICO, Julie. Science teachers' beliefs about the influence of their summer research experiences on their pedagogical practices. Journal of Science Teacher Education, Auburn, v. 24, n. 8, p. 1241-1261, 2013.

OSAMOR, Pauline; KASS, Nancy. Decision-making and motivation to participate in biomedical research in southwest Nigeria. Developing World Bioethics, E.E.U.U., v. 12, n. 2, p. 87-95, 2012.

RIP, Arie. The future of research universities. Prometheus, London, v. 29, n. 4, p. 443-453, 2011.

ROJAS, Mauricio. La actitud estudiantil sobre la investigación en la universidad: un estudio de caso en seis universidades de Colombia. Investigación \& Desarrollo, Barranquilla, v. 18, n. 2, p. 370-389, 2011.

RUSSELL, Susan; HANCOCK, Mary; MCCULLOUGH, James. Benefits of undergraduate research experiences. Science, Wasgington, v. 316, n. 5824, p. 548-549, 2007.

SCHWARZ, Rosângela; BORGES, Aline. Periódicos científicos na America Latina: títulos em acesso aberto indexados no ISI e Scopus. Perspectivas em Ciência da Informação, Belo Horizonte, v. 17, n. 4, p. 76-99, 2012.

SEYMOUR, Elaine et al. Establishing the benefits of research experiences for undergraduates in the sciences: first findings from a three-year study. Science Education, Boulder, v. 88, n. 4, p. 493-534, 2004.

TAMAYO, Mario; RESTREPO, María. Cultura investigativa en la Universidad. Santiago de Cali. Cali: Universidad Icesi, 2011. Disponible en: <http://hdl.handle.net/10906/5339>. Acceso en: 04 mar. 2015.

VILLAREJO, Merna et al. Encouraging minority undergraduates to choose science careers: career paths survey results. CBE-Life Sciences Education, E.E.U.U., v. 7, n. 4, p. 394-409, 2008.

WILLIS, David, KRUEGER, Paul; KENDRICK, Alice. The influence of a research experiences for undergraduates program on student perceptions and desire to attend graduate school. Journal of STEM Education, Auburn, v. 14, n. 2, p. 21 - 28, 2013.

WILSON, Anna et al. Academics' perceptions of the purpose of undergraduate research experiences in a research-intensive degree. Studies in Higher Education, Oxford, v. 37, n. 5, p. 513-526, 2012. 
WOODCOCK, Anna et al. Engineering students' beliefs about research: sex differences, personality, and career plans. Journal of Engineering Education, Washington, v. 101, n. 3, p. 495-511, 2012.

ZIMBA, Horácio; MUELLER, Suzana. Colaboração internacional e visibilidade científica de países em desenvolvimento: 0 caso da pesquisa na área de medicina veterinária em Moçambique. Informação \& Sociedade: Estudos, João Pessoa, v. 14, n. 1, p. 4568, 2004.

Recibido en: 23.05.2015

Aprobado en: 15.03.2016

Alejandro Valencia-Arias es $\mathrm{PhD}(\mathrm{c})$, magister en Ingeniería de Sistemas e Ingeniero Administrador. Investigador en el grupo de Ciencias Administrativas del Instituto Tecnológico Metropolitano. Entre sus líneas de investigación se encuentran: educación superior, mobile learning y emprendimiento.

Daniela Morales es Ingeniera Industrial e Ingeniera Administradora de la Universidad Nacional de Colombia. Investiga en temáticas de mercados financieros y de educación superior.

Laura Vanegas es Ingeniera Administradora de la Universidad Nacional de Colombia, profesora asistente de la Facultad de Ingeniería de la Universidad de los Andes. Se dedica a la investigación cualitativa y cuantitativa de problemáticas relacionadas con educación y con el medio ambiente.

Martha Luz Benjumea es magister en Ingeniería Administrativa y especialista en Gerencia Organizacional. Docente e investigadora del grupo en Ciencias Administrativas del Instituto Tecnológico Metropolitano (ITM) en temas de prospectiva y emprendimiento. 